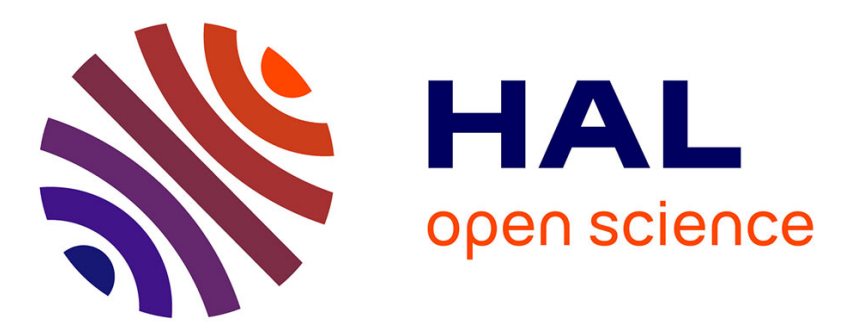

\title{
Partial linearization of the PVTOL aircraft with internal stability
}

Vincent Léchappé, Yannick Aoustin, L. Marquez Martinez, Claude Moog

\section{To cite this version:}

Vincent Léchappé, Yannick Aoustin, L. Marquez Martinez, Claude Moog. Partial linearization of the PVTOL aircraft with internal stability. 52nd Conference on Decision and Control, Dec 2013, Florence, Italy. 10.1109/CDC.2013.6760268 . hal-00858102

\section{HAL Id: hal-00858102 https://hal.science/hal-00858102}

Submitted on 25 Jan 2016

HAL is a multi-disciplinary open access archive for the deposit and dissemination of scientific research documents, whether they are published or not. The documents may come from teaching and research institutions in France or abroad, or from public or private research centers.
L'archive ouverte pluridisciplinaire HAL, est destinée au dépôt et à la diffusion de documents scientifiques de niveau recherche, publiés ou non, émanant des établissements d'enseignement et de recherche français ou étrangers, des laboratoires publics ou privés. 


\title{
Partial linearization of the PVTOL aircraft with internal stability
}

\author{
V. Léchappé, Y. Aoustin, L.A. Márquez-Martínez and C.H. Moog
}

\begin{abstract}
The planar vertical take-off and landing (PVTOL) aircraft is worked out by inspecting the various feedback linearization solutions. Partial linearizations of the state space are performed, which ensure the stability of the associated internal dynamics. The results are obtained through the search of dummy outputs with a 0-dimensional, 1-dimensional or 2dimensional zero-dynamics that are stable.

Index Terms-nonlinear, MIMO, underactuated, nonminimum phase, zero-dynamics, internal dynamics, algebraic method, partial linearization, stabilization, PVTOL
\end{abstract}

\section{INTRODUCTION}

VTOL (Vertical Take-Off and Landing) aircrafts are adapted for applications in a narrow environment since they do not require strips for take-off or landing. Helicopters and quad-rotors are examples of such devices. PVTOL (Planar VTOL) aircrafts are VTOL aircrafts that perform only in the vertical plane. Hauser et al. [1] have exhibited the mechanical model of the PVTOL in 1992. It is obtained by applying Newton's laws of motion to a rigid body subjected to its weight and thrusts (see [2] for a set of equations). The model is simplified because it does not take into account aerodynamic effects, wings deformation and propellers dynamics (for more complete models see [3] and [4]). This model motivated numerous works as it keeps the main characteristics of 6DOF VTOL vehicles, including the coupling between rotation and translation. Furthermore, it is simple enough for the purpose of control. This coupling is represented by the parameter $\epsilon$ in (1). It is shown in [1] that there exists an unstable internal dynamics of order 2 when the "natural" outputs (horizontal position and altitude) are linearized by state feedback. The system is said to be non-minimum phase. A controller was designed neglecting the coupling, and applied to the real system. Good results are obtained for slightly non-minimum phase systems (with weak coupling). The main drawback remains when the coupling becomes stronger.

Besides, many papers have considered the control of the PVTOL using various methods. Amongst them, one may cite digital control [5], flat control [6], backstepping [7][8], sliding mode control [9], predictive control [10] and modelfree control [2]. Various control problems have been considered like output tracking [11], stabilization with bounded inputs [12], robustness to delay in the inputs [13], robustness to delayed outputs [14], robustness to crosswind [15] and robustness to aerodynamic effects [4].

Yannick Aoustin, Vincent Léchappé and Claude H. Moog are with L'UNAM, IRCCyN, UMR CNRS 6597, Ecole Centrale de Nantes, Université de Nantes, France Firstname.Namedirccyn.ec-nantes.fr. Luis Alejandro Márquez Mártinez is with CICESE Research Center Ensenada B. C. Mexico, Imarquez@cicese.mx
In [6], flat outputs are computed to completely linearize the system. Those flat outputs are interpreted as the coordinates of the center of percussion. The controller yields a good performance for both slight and strong couplings but exhibits a singularity. Besides, it requires the computations of the fourth time derivatives of each output. Other authors use linearization but not on the entire system [16] or just to shape the model before applying other methods [17]. In [16], Consolini et al. design a feedback that decomposes the PVTOL model into a transversal subsystem which is linear time invariant and a tangential subsystem. These subsystems are then driven by transversal and tangential control inputs to solve a path following problem. The methodology exhibits output invariance of the path and boundedness of the roll angle.

An exhaustive analysis of the feedback linearization solutions is not available yet: the two major milestones are [1], which copes with a 2-dimensional unstable zero-dynamics, and [6] which gets rid of any zero-dynamics. The present article investigates new solutions: two outputs are sought such that the resulting zero-dynamics has dimension 1. It is shown that they can be computed so that their zero-dynamics is asymptotically stable, and its velocity can be tuned as well (by the proper choice of the outputs).

This paper is organized as follows: Section II is a presentation and a structural analysis of the model and the existing results [1], [6]. We will also explain some results on the order of the zeros at infinity. Section III is divided in two parts: the first one computes the inversion algorithm and displays controller's equations. The second one is dedicated to the internal dynamics stability. Finally, several simulation results are given and the performance is compared with the one derived from the flat outputs linearization [6] in section IV.

\section{PRESENTATION AND STRUCTURAL ANALYSiS With THE ALGEBRAIC METHOD}

\section{A. Analysis of the PVTOL equations}

The normalized equations (1) of the PVTOL, associated to Figure 1, were obtained in [1], considering the aircraft as a rigid body upon which act its weight and the thrusts:

$$
\left\{\begin{array}{l}
\ddot{x}=-u_{1} \sin \theta+\epsilon u_{2} \cos \theta \\
\ddot{z}=u_{1} \cos \theta+\epsilon u_{2} \sin \theta-1 \\
\ddot{\theta}=u_{2}
\end{array}\right.
$$

where $\epsilon>0 . x$ and $z$ denote, respectively, the horizontal position and the altitude of the PVTOL's center of gravity and $\theta$ the roll angle. $u_{1}$ is the thrust which is always positive and $u_{2}$ is the rolling moment."-1" is the normalized gravity 


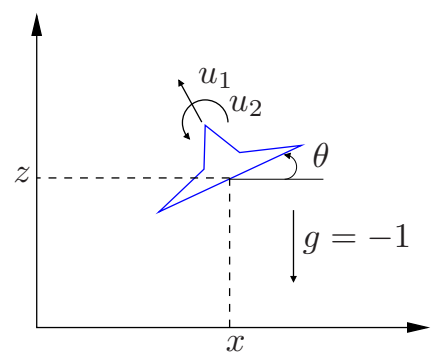

Fig. 1. View of the PVTOL aircraft in the vertical plane

acceleration, and the parameter $\epsilon$ represents the coupling between the horizontal and the vertical motions. The 6-

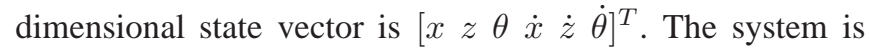
underactuated because it has three degrees of freedom $(x, z, \theta)$ but only two control inputs $\left(u_{1}, u_{2}\right)$.

We are now going to analyze $\mathcal{H}_{k}$ subspaces to characterize outputs with the maximum relative degree. $\mathcal{H}_{k}$ are subspaces containing all the elements (one-forms) whose relative degree is greater than or equal to $k$. More explanations over $\mathcal{H}_{k}$ subspaces and integrability condition are available in [18]. After some computations, we obtain:

$$
\begin{aligned}
& \mathcal{H}_{1}=\operatorname{span}\{d x, d \dot{x}, d z, d \dot{z}, d \theta, d \dot{\theta}\} \\
& \mathcal{H}_{2}=\operatorname{span}\{d x, d z, d \theta, d(\cos \theta \dot{x}+\sin \theta \dot{z}-\epsilon \dot{\theta})\} \\
& \mathcal{H}_{3}=\operatorname{span}\left\{\omega_{1}, \omega_{2}\right\} \\
& \mathcal{H}_{4}=\operatorname{span}\{0\} \\
& \text { with } \omega_{1}=\cos \theta d x+\sin \theta d z-\epsilon d \theta \\
& \text { and } \omega_{2}=\dot{\theta} \sin \theta d x+\cos \theta d \dot{x}-\dot{\theta} \cos \theta d z+\sin \theta d \dot{z}-\epsilon d \dot{\theta}
\end{aligned}
$$

$\mathcal{H}_{4}$ equal to 0 means that there is no autonomous element so the system is accessible. However, $\mathcal{H}_{3}$ is not fully integrable according to Frobenius Theorem because $d \omega_{1} \wedge \omega_{1} \wedge \omega_{2} \neq 0$ so the input/state linearization can not be solved by static feedback. Finally, it can be shown that there is no (nonzero) exact one-form in $\mathcal{H}_{3}$ : no output exists with a relative degree greater than or equal to 3 .

\section{B. Partial linearization with a 2-dimensional internal dy- namics [1]}

Choosing $x$ and $z$ as outputs [1], the following decoupling matrix is obtained:

$$
\left(\begin{array}{ll}
\frac{\partial \ddot{x}}{\partial u_{1}} & \frac{\partial \ddot{x}}{\partial u_{2}} \\
\frac{\partial \ddot{z}}{\partial u_{1}} & \frac{\partial \ddot{z}}{\partial u_{2}}
\end{array}\right)=\left(\begin{array}{cc}
-\sin \theta & \epsilon \cos \theta \\
\cos \theta & \epsilon \sin \theta
\end{array}\right)
$$

whose determinant is $-\epsilon$. Since $\epsilon \neq 0$ the matrix rank is 2 and it is said that the outputs ( $x$ and $z$ ) have 2 zeros at infinity of order 2. The notion of zeros at infinity describes how control inputs occur explicitly in output derivatives. Here $u_{1}$ and $u_{2}$ appear independently in the second derivative of $x$ and $z$ since the decoupling matrix is invertible. Applying the static feedback:

$$
\left(\begin{array}{l}
u_{1} \\
u_{2}
\end{array}\right)=\left(\begin{array}{cc}
-\sin \theta & \cos \theta \\
\frac{\cos \theta}{\epsilon} & \frac{\sin \theta}{\epsilon}
\end{array}\right)\left(\left(\begin{array}{l}
v_{1} \\
v_{2}
\end{array}\right)+\left(\begin{array}{l}
0 \\
1
\end{array}\right)\right)
$$

leads to the system :

$$
\left\{\begin{array}{l}
\ddot{x}=v_{1} \\
\ddot{z}=v_{2} \\
\ddot{\theta}=\frac{1}{\epsilon}\left(\sin \theta+v_{1} \cos \theta+v_{2} \sin \theta\right)
\end{array}\right.
$$

We see that the evolution of the third equation is imposed by the decoupling of $\ddot{x}$ and $\ddot{z}$ : this is the internal dynamics associated to the outputs $x$ and $z$. The order of the dynamics is calculated subtracting the number of states from the sum of the order of the zeros at infinity: we have an internal dynamics of order $6-4=2$. Canceling the outputs, and all their time derivatives, the zero-dynamics is obtained as:

$$
\ddot{\theta}=\frac{1}{\epsilon} \sin \theta
$$

Equation (2) is the equation of an undamped pendulum which has two equilibrium points. However, one equilibrium point is unstable so it is said that the system is non-minimum phase for the outputs $x$ and $z$.

\section{Flat outputs [6]}

In [6], it has been shown that the outputs

$$
x_{f}=x-\epsilon \sin \theta \quad z_{f}=z+\epsilon \cos \theta
$$

are without zero-dynamics. They are called flat outputs. By computing the second time derivative of $x_{f}$, and the fourth time derivative of $z_{f}$, we get the invertible decoupling matrix:

$$
\left(\begin{array}{cc}
\frac{\partial \ddot{x}_{f}}{\partial u_{1}} & \frac{\partial \ddot{x}_{f}}{\partial u_{2}} \\
\frac{\partial z_{f}^{(4)}}{\partial u_{1}} & \frac{\partial z_{f}^{(4)}}{\partial u_{2}}
\end{array}\right)=\left(\begin{array}{cc}
-\sin \theta & 0 \\
0 & -\eta_{1} \sin \theta
\end{array}\right)
$$

where $\eta_{1}=u_{1}-\epsilon \dot{\theta}^{2}$. Thus, the orders of zeros at infinity are 2 and 4 , and the sum is 6 as the number of states so the internal dynamics is empty. The dynamic feedback

$$
\left\{\begin{array}{l}
\dot{\eta}_{1}=\eta_{2} \\
\dot{\eta}_{2}=-v_{1} \sin \theta+v_{2} \cos \theta+\eta_{1} \dot{\theta}^{2} \\
u_{1}=\eta_{1}+\epsilon \dot{\theta}^{2} \\
u_{2}=\frac{1}{\eta_{1}}\left(-v_{1} \cos \theta-v_{2} \sin \theta-2 \eta_{2} \dot{\theta}\right)
\end{array}\right.
$$

where $\eta_{2}=\dot{\eta}_{1}$, decouples and fully linearizes PVTOL equations:

$$
\left\{\begin{array}{l}
x_{f}^{(4)}=v_{1} \\
z_{f}^{(4)}=v_{2}
\end{array}\right.
$$

Note that the controller dynamics is $2\left(\eta_{1}, \eta_{2}\right)$ and, that it has a singularity in $\eta_{1}=0$. Then by an appropriate poleplacement, $x_{f}$ and $z_{f}$ are stabilized. That is equivalent to stabilize $x$ and $z+\epsilon$ because $\theta=0$ at equilibrium. The result holds even for strong coupling values. 
D. Partial linearization with a 1 dimension internal dynamics

The exhaustive investigation of partial linearization is not done yet, although new alternatives are offered to circumvent the minimum phase issue, as shown next. On one hand, the outputs $(x, z)$ were recalled to have zeros at infinity of order $(2,2)$, and an internal dynamics of order 2 . On the other hand, the outputs $\left(x_{f}, z_{f}\right)$ have zeros at infinity of order $(4,2)$, and an internal dynamics of order 0 . Any alternative choice of output will feature a structure displayed in the following table:

\begin{tabular}{cc}
\hline $\begin{array}{c}\text { Orders of the zeros at } \\
\text { infinity }\end{array}$ & $\begin{array}{c}\text { Order of the internal } \\
\text { dynamics }\end{array}$ \\
\hline$(5,1)(4,2)(3,3)$ & 0 \\
$(4,1)(3,2)$ & 1 \\
$(2,2)(3,1)$ & 2 \\
$(2,1)$ & 3 \\
$(1,1)$ & 4 \\
\hline
\end{tabular}

The expression " $\left(n_{1}, n_{2}\right)$ structure" is used to designate outputs with "zeros at infinity of order $\left(n_{1}, n_{2}\right)$ ". As a consequence, it is equivalent to say that outputs have a $\left(n_{1}, n_{2}\right)$ structure or a $\left(n_{2}, n_{1}\right)$ structure.

Let us explain why we focus on outputs with $(4,1)$ structure. First, we did not consider the cases where the order of internal dynamics is larger than 1 because we obviously want to control the larger part of the system. $(5,1)$ structure would lead to a dynamic controller of dimension greater than or equal to 3 which would slow down the response. Structure $(3,3)$ looks interesting because it would guarantee a static controller. However, it cannot be achieved since it would require outputs with a relative degree of 3 which do not exist as we have seen in section II-A. Structure $(4,2)$ has already been studied. The structure $(3,2)$ yields outputs with undesired singularities [12]. For example, the outputs

$$
x_{(3,2)}=\theta \quad y_{(3,2)}=x \cos \theta+z \sin \theta-\epsilon \theta
$$

have a $(3,2)$ structure because the decoupling matrix is

$$
\left(\begin{array}{ll}
\frac{\partial \ddot{x}_{(3,2)}}{\partial u_{1}} & \frac{\partial \ddot{x}_{(3,2)}}{\partial u_{2}} \\
\frac{\partial \dddot{y}_{(3,2)}}{\partial u_{1}} & \frac{\partial \dddot{y}_{(3,2)}}{\partial u_{2}}
\end{array}\right)=\left(\begin{array}{cc}
0 & 1 \\
2 \dot{\theta} & 0
\end{array}\right)
$$

The inversion yields $u_{1}=\frac{1}{2 \dot{\theta}}(\ldots)$ but $\dot{\theta}$ has to be 0 at the equilibrium so we necessary go through this singularity. Next, the structure $(4,1)$ is investigated extensively, with the outputs $\left(y_{1}, y_{2}\right)$ which are derived from the flat outputs as follows:

$$
y_{1}=x_{f} \quad y_{2}=z_{f}+k \dot{z}_{f}-\epsilon
$$

where $k$ is a real non-zero constant. This parameter $k$ is introduced to tune the internal dynamics stability and velocity. In the following, we are going to find out dynamic controller's equations, and show that we can tune the stability and velocity of the zero-dynamics thanks to $k$.
III. OutPuts With ZEROS AT INFINITY OF ORDER $(4,1)$

\section{A. Inversion algorithm and controller's equations}

Consider the following outputs:

$$
\begin{gathered}
y_{1}=x-\epsilon \sin \theta \\
y_{2}=z+\epsilon \cos \theta+k \dot{z}-k \epsilon \dot{\theta} \sin \theta-\epsilon
\end{gathered}
$$

The inversion algorithm is a way to know if the system is invertible, and if this is the case, to derive the controller's equations. This process of inversion is obviously related to the chosen outputs. The general algorithm is developed in [18]. Let us apply it to the PVTOL with the outputs $y_{1}$ and $y_{2}$ :

$$
\begin{gathered}
\dot{y}_{1}=\dot{x}-\epsilon \dot{\theta} \cos \theta \\
\dot{y}_{2}=\dot{z}-\epsilon \dot{\theta} \sin \theta+k u_{1} \cos \theta-k-k \epsilon \dot{\theta}^{2} \cos \theta
\end{gathered}
$$

We notice that the control appears only in $\dot{y}_{2}$, and we cannot fully invert the system. There is a zero at infinity of order one. Consequently, we carry on derivating $y_{1}$ :

$$
\ddot{y}_{1}=-u_{1} \sin \theta+\epsilon \dot{\theta}^{2} \sin \theta
$$

This time $u_{1}$ appears in $\ddot{y}_{1}$ but the decoupling matrix has still no inverse. At this point, we see that linearization by static feedback is impossible, and we are going to introduce again $\eta_{1}=u_{1}-\epsilon \dot{\theta}^{2}$ as a new state to delay the apparition of $u_{1}$ : we are designing a dynamic feedback. From (7) we have:

$$
\ddot{y}_{1}=-\eta_{1} \sin \theta
$$

Remark that following explicitly the inversion algorithm from [18], $\dot{y}_{2}$ would have been introduced instead of $\eta_{1}$. Nevertheless, it has been prefered to choose $\eta_{1}$ as the controller dynamics (as in [6] for flat outputs) because it makes computations easier, and avoids to add an extra singularity. See [19] for more details about the construction of such decoupling feedbacks. We continue differentiating $y_{1}$ but $u_{2}$ still does not explicitly appear:

$$
\dddot{y}_{1}=-\dot{\theta} \eta_{1} \cos \theta-\dot{\eta}_{1} \sin \theta
$$

so we differentiate once more, and we obtain:

$y_{1}^{(4)}=-u_{2} \eta_{1} \cos \theta+\dot{\theta}^{2} \eta_{1} \sin \theta-\dot{\theta} \eta_{1} \cos \theta-\dot{\theta} \dot{\eta}_{1} \cos \theta-\ddot{\eta}_{1} \sin \theta$

and the decoupling matrix is:

$$
\left(\begin{array}{cc}
\frac{\partial \dot{y}_{2}}{\partial u_{1}} & \frac{\partial \dot{y}_{2}}{\partial u_{2}} \\
\frac{\partial y_{1}^{(4)}}{\partial u_{1}} & \frac{\partial y_{1}^{(4)}}{\partial u_{2}}
\end{array}\right)=\left(\begin{array}{cc}
k \cos \theta & 0 \\
0 & -\eta_{1} \cos \theta
\end{array}\right)
$$

This time it is invertible since the determinant is non zero. There is a zero at infinity of order 4 . Now, we are able to decouple and linearize the PVTOL as follows:

$$
\left\{\begin{array}{l}
y_{1}^{(4)}=v_{1} \\
y_{2}^{(3)}=v_{2}
\end{array}\right.
$$


with the controller defined by equations :

$$
\left\{\begin{array}{l}
\dot{\eta_{1}}=\eta_{2} \\
\dot{\eta_{2}}=\frac{1}{k}\left[-k v_{1} \sin \theta-v_{2} \cos \theta+(1-k) \dot{\theta} \eta_{1} \cos \theta \sin \theta+\right. \\
\left.k \dot{\theta} \eta_{1}+k \dot{\theta} \eta_{2} \cos \theta \sin \theta-\eta_{2} \cos ^{2} \theta\right] \\
u_{1}=\eta_{1}+\epsilon \dot{\theta}^{2} \\
u_{2}=\frac{1}{k \eta_{1}}\left[-k v_{1} \cos \theta-v_{2} \sin \theta-\dot{\theta} \eta_{1}\left(\sin ^{2} \theta+k \cos ^{2} \theta\right)\right. \\
\left.+\eta_{2} \sin \theta \cos \theta-k \dot{\theta} \eta_{2}\left(\cos ^{2} \theta+2 \sin ^{2} \theta\right)\right]
\end{array}\right.
$$

The controller exhibits the same singularity as the controller for flat outputs : $\eta_{1}=0$. It also has an internal dynamics of order $2\left(\eta_{1}, \eta_{2}\right)$. This dynamics has not to be confused with the internal dynamics of the PVTOL (called $\sigma$ in the next parts) which is of dimension 1 for these outputs. System (11) is linear, and can be stabilized by pole placement. The objective of the next paragraph is to demonstrate that for some values of $k$ we can stabilize system (1). For that purpose, a suitable design of the zero-dynamics was achieved.

\section{B. Zero-dynamics stability}

$y_{1}$ and $y_{2}$ have, respectively, zeros at infinity of order 1 and 4 , so the system is only partially linearized and there remains an internal dynamics of order 1 . In this section, the zero-dynamics is computed, and its stability is studied. Let us call $\sigma$ the internal dynamics. The coordinate $\sigma$ represents the internal dynamics of system (1) (with outputs $y_{1}$ and $\left.y_{2}\right)$ if and only if there exists a local diffeomorphism $\phi$ between $X_{s}=\left(x, \dot{x}, z, \dot{z}, \theta, \dot{\theta}, \eta_{1}, \eta_{2}\right)$ and $\zeta=$ $\left(y_{1}, \dot{y}_{1}, \ddot{y}_{1}, y_{1}^{(3)}, y_{2}, \dot{y}_{2}, \ddot{y}_{2}, \sigma\right)$. There are a lot of solutions so to make the computations easier, we choose to take $\sigma$ of relative degree greater or equal to 2 . With this condition, we have limited the possibilities without losing generality because we are actually looking for the zero-dynamics and not the internal dynamics. Even if there are plenty of internal dynamics, there is only one zero-dynamics associated with these outputs. After some computations, we notice that $z$ complies with these two constraints so we now consider $\sigma=z$. The objective is to express $\dot{z}$ as a function of $\zeta=\left(y_{1}, \dot{y}_{1}, \ddot{y}_{1}, y_{1}^{(3)}, y_{2}, \dot{y}_{2}, \ddot{y}_{2}, z\right)$, and then to cancel outputs and their derivatives to exhibit the equation of the zerodynamics. This the classical way to find the expression of a zero-dynamics. In our case, it is easier to show first that when zero-dynamics is reached, $\theta$ must be equal to zero. This result is demonstrated by a contradiction proof :

Let's suppose that $\exists t_{0}$ such that $\theta\left(t_{0}\right) \neq 0$ (modulo $\pi$ ).

When the outputs and their derivatives are cancelled, equation (8) becomes $0=-\sin \theta\left(t_{0}\right) \eta_{1}\left(t_{0}\right)$ and it follows that

$$
\eta_{1}\left(t_{0}\right)=0
$$

Using this equality in (9) results in $0=-\sin \theta\left(t_{0}\right) \dot{\eta}_{1}$ so

$$
\dot{\eta}_{1}\left(t_{0}\right)=0
$$

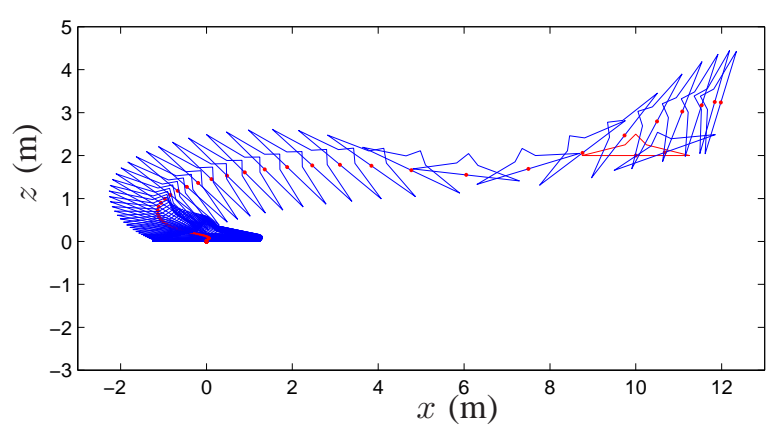

Fig. 2. Aggressive maneuver with strong coupling : $\epsilon=2$

Finally, the contradiction $0=-1$ is found by substituting (12) and (13) in

$$
\ddot{y}_{2}=\eta_{1} \cos \theta-k \dot{\theta} \eta_{1} \sin \theta+k \dot{\eta}_{1} \cos \theta-1
$$

The negation of the initial claim leads to desired result :

$$
\theta=0 \quad(\operatorname{modul} \pi)
$$

when zero-dynamics is reached. A similar argument gives

$$
\dot{\theta}=0
$$

From (3) and (14), we know that $x$ is always stabilized independently of the value of the constant k. From (4) and (14), we draw the zero-dynamics equation:

$$
\dot{z}+\frac{1}{k} z=0
$$

This is a simple linear ODE of order 1 with constant coefficients.

- $k>0$ : (16) is asymptotically stable.

- $k<0$ : (16) is unstable.

This result was expected regarding the definition of $y_{2}: y_{2}=$ $z_{f}+k \dot{z}_{f}-\epsilon$. Indeed, stabilizing $y_{2}$ implies $z_{f}+k \dot{z}_{f}=\epsilon$ so the sign of the constant $k$ governs the stability of $z_{f}$ and consequently the stability of $z$. If the internal dynamics $z$ is stable, we see from (5) and (15) that $\dot{x}$ also tends to zero. To sum up, $\left(y_{1}, \dot{y}_{1}, \ddot{y}_{1}, y_{1}^{(3)}, y_{2}, \eta_{1}, \eta_{2}\right) \rightarrow \mathbf{0}_{1 \times 7}$, and $k>0$ results in $(x, \dot{x}, z, \dot{z}, \theta, \dot{\theta}) \rightarrow \mathbf{0}_{1 \times 6}$. It means that stabilizing (11), and choosing $k>0$ induces the stabilization of system (1) to the origin.

\section{Simulation AND COMPARISON With FLAT OUTPUTS}

Simulations were carried out to check the controller efficiency for a large coupling parameter. We set $\epsilon=2$, $k=0.1$, and an aggressive maneuver is imposed. The result is displayed on Figure 2. The initial position of the aircraft is in red. We observe the non-minimum phase character of outputs $x$ and $z$ because the $x$ response begins in the opposite direction. Besides, the controller behaves well even for a large value of $\epsilon$. This is important because the first studies did not tolerate large coupling parameters since they were synthesized on an approximate model $(\epsilon=0)$ [1] and [7]. The controller has also been checked to be robust with respect to variations of $\epsilon$ : for $\epsilon$ values around 1, the system is stabilized even for deviations up to $50 \%$. Figures 3, 4, and 5 show the influence of parameter $\mathrm{k}$ on the stabilization. 
For these simulations, we chose $\epsilon=0.5, x_{0}=10, z_{0}=$ 2 and other initial conditions are in 0 . We notice that

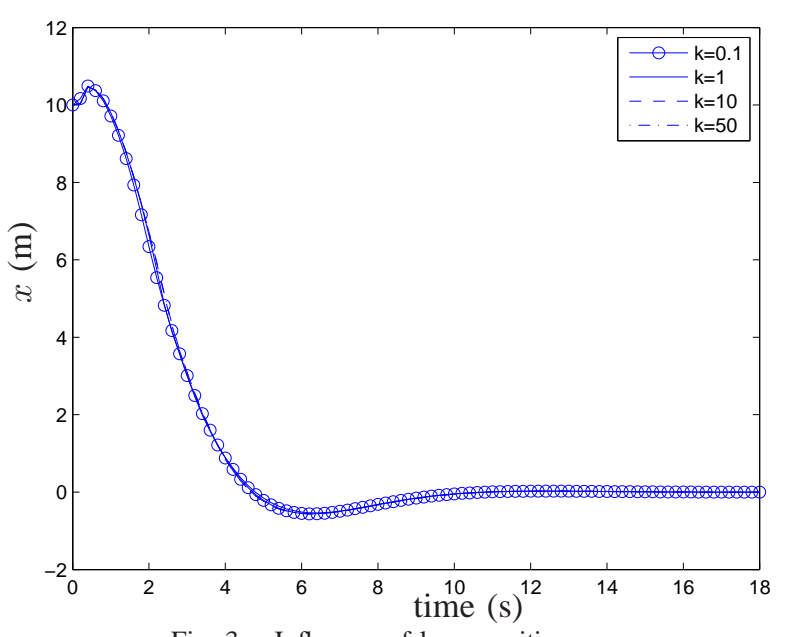

Fig. 3. Influence of $\mathrm{k}$ on position $\mathrm{x}$

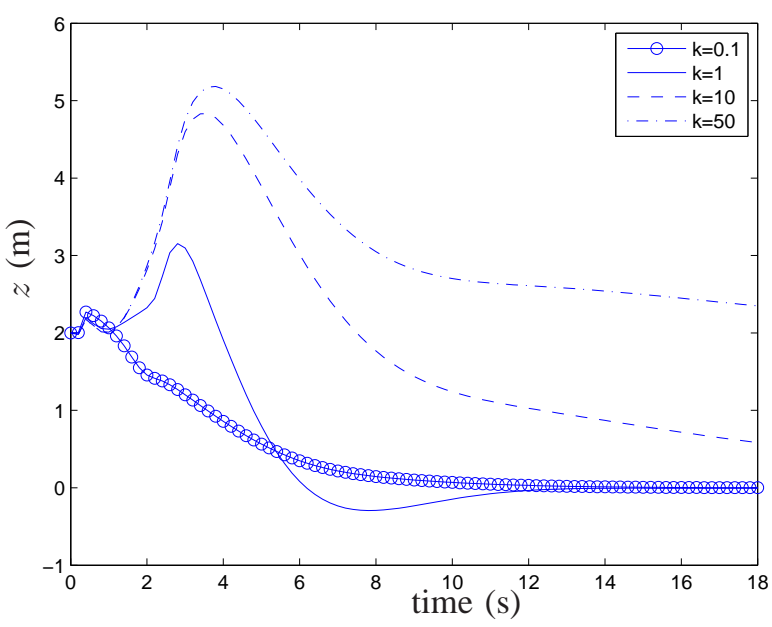

Fig. 4. Influence of $\mathrm{k}$ on altitude $\mathrm{z}$

parameter $k$ has little influence on $x$ : all controllers have approximately the same response time. This is coherent since $k$ does not appear in $y_{1}$. On the contrary, the higher $k$, the higher the response time for $z$ : this confirms the result (16). This is interesting to notice that a modification on $k$ has almost no influence on the $x$ dynamics but strongly affects $z$ dynamics. It is the $\theta$ dynamics which is modified and cause the slowing down or the acceleration of the $z$ dynamics. This is really useful to be able to control precisely the $z$ dynamics of an aircraft to take off and land properly. That is why we chose $\left(y_{1}, y_{2}\right)=\left(x_{f}, z_{f}+k \dot{z}_{f}-\epsilon\right)$ and not $\left(y_{1}, y_{2}\right)=\left(z_{f}, x_{f}+k \dot{x}_{f}-\epsilon\right)$ which would have lead to a similar zero-dynamics but in $x$. With these graphs, we cannot analyze precisely the response time because equation (16) is valid only when $y_{1}$ and $y_{2}$ are stabilized to zero. For all these responses we make sure that commands $u_{1}$ and $u_{2}$ do not go through singularities and keep low level. We also make sure that $u_{1}$ remains positive because it represents the thrust. On Figures 6 and 7, the controller in Section III (with $k=0.1$ and $k=1$ ) is compared with the one

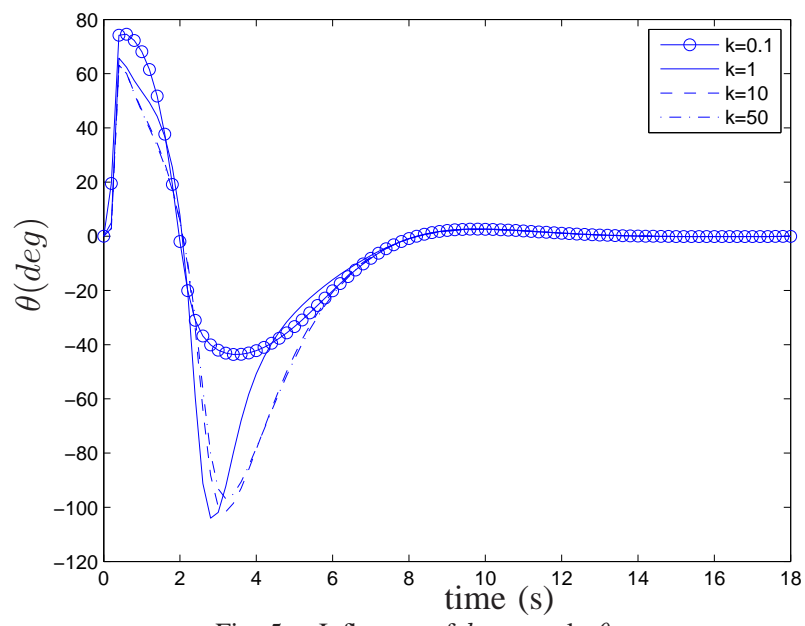

Fig. 5. Influence of $k$ on angle $\theta$

derived from flat outputs. The same pole placement is used for both controllers. Figure 6 confirms that $x$ dynamics is similar for the three controllers because $y_{1}$ does not depend on $k$ and $y_{1}=x_{f}$. Figure 7 shows that for $k=0.1$, the controller designed from the outputs with the structure $(4,1)$ is faster than the controller from flat outputs. This advantage is accentuated because it can be seen on Figures 8 and 9 that it requires less effort to achieve better result. The difference is clear for $u_{1}$ but it is still more important for $u_{2}$ where the "flat" controller needs four times more energy than " $(4,1) "$ controller. Note that time scale on Figures 8 and 9 has been voluntary reduced to focus on the beginning of the maneuver where the variations are the most important.

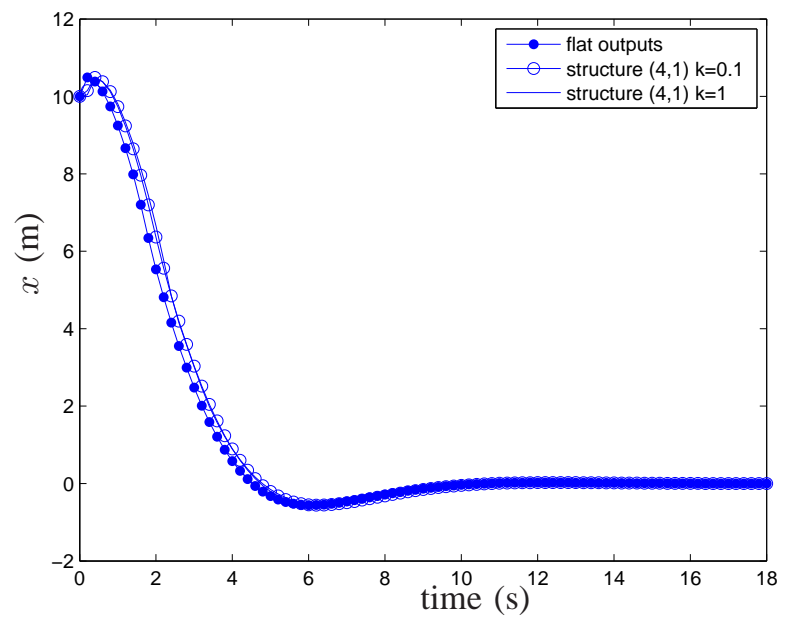

Fig. 6. Comparison between controllers derived from outputs with structure $(4,1)$ and from flat outputs: position $x$

\section{CONCLUSION}

In this paper, outputs have been computed with a structure at infinity $(4,1)$ that partially linearize the PVTOL aircraft. The resulting zero-dynamics was computed, and we have demonstrated that the stability was governed by the sign of $k$ and that the convergence speed was governed by the value of $k$. Simulations results are in accordance with our assertions, and have shown that the controller stabilizes the aircraft 


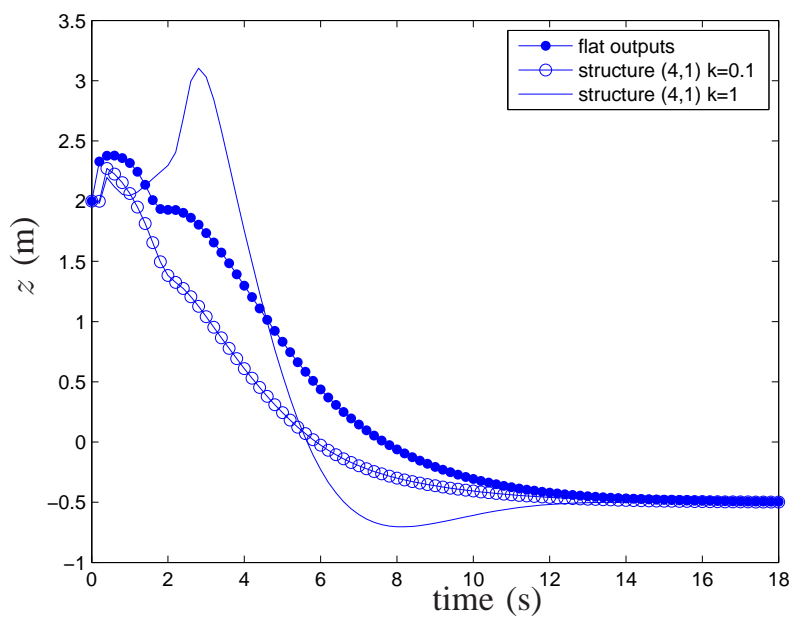

Fig. 7. Comparison between controllers derived from outputs with structure $(4,1)$ and from flat outputs: altitude $z$

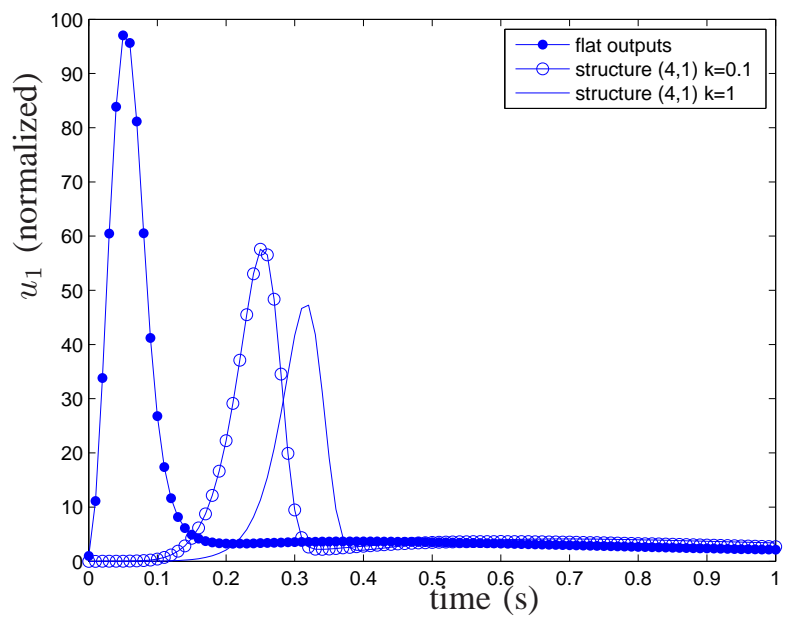

Fig. 8. Influence of $k$ on command $u_{1}$

even with a strong input coupling. Furthermore, for small values of $k$, the " $(4,1)$ " controller has better performance for $z$ dynamics with lower input requirements. Finally, results are achieved with the possibility to adjust $z$ dynamics. More generally, a new perspective for dealing with zero-dynamics is offered for general underactuated systems.

\section{ACKNOWLEDGEMENTS}

The authors would like to thank an anonymous reviewer for his suggestion that allowed to avoid some singularities in the construction of the " $(4,1)$ " compensator.

\section{REFERENCES}

[1] J. Hauser, S. Sastry, and G. Meyer, "Nonlinear control design for slightly non-minimum phase systems : applications to v/stol aircraft," Automatica (Oxford), vol. 28, no. 4, pp. 665-679, 1992.

[2] S. Riachy, M. Fliess, C. Join, and J.-P. Barbot, "Vers une simplification de la commande non linéaire : l'exemple d'un avion à décollage vertical," in 6e Conférence Internationale Francophone d'Automatique, CIFA 2010, Nancy, France, Jun. 2010, p. CDROM. [Online]. Available: http://hal.inria.fr/inria-00463605

[3] R. Cardenas and L. T. Aguilar, "Output feedback sliding mode control of a pvtol including actuators dynamics," in Proc. of the IEEE International Conference on Control Applications (CCA), sept 2011, pp. $1482-1486$.

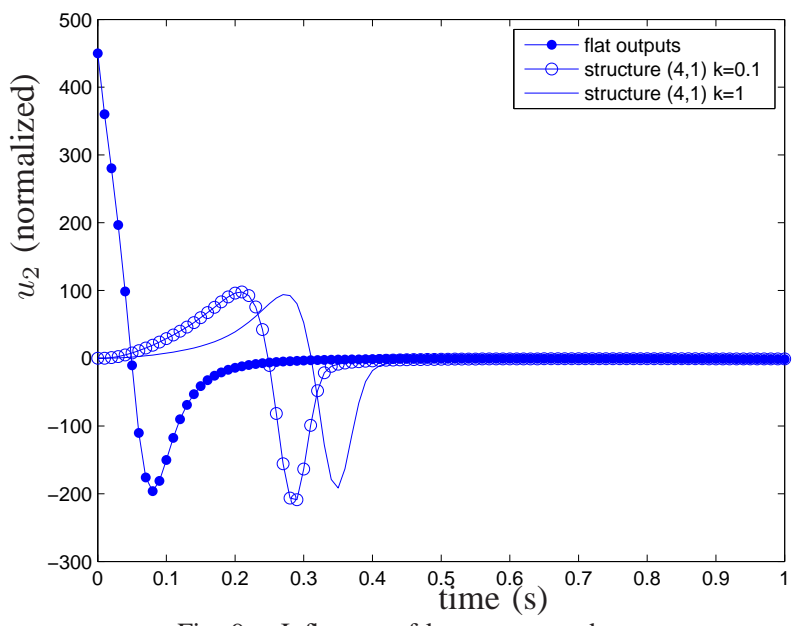

Fig. 9. Influence of $\mathrm{k}$ on command $u_{2}$

[4] R. Wood, B. Cazzolato, and D. Halim, "A global nonlinear control design for a pvtol vehicle with aerodynamics," in Proc. of the 44th IEEE Conference on Decision and Control and European Control Conference. CDC-ECC '05, dec 2005, pp. $7478-7484$.

[5] P. Di Giamberardino and M. Djemai, "Digital control of a pvtol aircraft," in Proc of the 33rd Conference on Decision and Control, vol. 4, dec 1994, pp. $3844-3849$.

[6] P. Martin, S. Devasia, and B. Paden, "A different look at output tracking: control of a vtol aircraft," in Proc. of the 33rd IEEE Conference on Decision and Control, vol. 3, dec 1994, pp. 2376 2381.

[7] R. Sepulchre, M. Jankovic, and P. Kokotovic, Constructive Nonlinear Control. Springer-Verlag, Berlin, Heidelberg, New-York, 1997.

[8] R. Olfati-Saber, "Global configuration stabilization for the vtol aircraft with strong input coupling," IEEE Trans. on Automatic Control, vol. 47, no. 11, pp. 1949 - 1952, nov 2002.

[9] X.-Y. Lu, S. K. Spurgeon, and I. Postxethwaite, "Robust variable structure control of a pvtol aircraft," International Journal of Systems Science, vol. 28, no. 6, pp. 547-558, 1997.

[10] A. Chemori and N. Marchand, "A prediction based nonlinear controller for stabilization of a non-minimum phase PVTOL aircraft," International Journal of Robust and Nonlinear Control, vol. 18, pp. 876-889, 2008. [Online]. Available: http://hallirmm.ccsd.cnrs.fr/lirmm-00305312

[11] A. Gruszka, M. Malisoff, and F. Mazenc, "On tracking for the pvtol model with bounded feedbacks," in Proc. of the American Control Conference, 29 2011-july 12011 , pp. $1428-1433$.

[12] V. Léchappé, "Analyse et commande du pvtol par la méthode algébrique," Master's thesis, Ecole Centrale de Nantes, 2012.

[13] R. Francisco, F. Mazenc, and S. Mondié, Global asymptotic stabilization of a PVTOL aircraft model with delay in the input, L. N. i. C. Springer-Verlag and I. Sciences, Eds., 2007, vol. 352.

[14] X. .Wang, J. .Liu, and K. .Cai, "Tracking control for a velocity sensorless VTOL aircraft with delayed outputs," Automatica, pp. 2876-2882, 2009.

[15] L. Muñoz, O. .Santos, and P. .Castillo, in Proc. of the IEEE/RSJ International Conference on Intelligent Robots and Systems, Tapei, Taiwan, sep 2010, pp. 1606 - 1611.

[16] L. Consolini, M. Maggiore, C. Nielsen, and M. Tosques, "Path following for the \{PVTOL\} aircraft," Automatica, vol. 46, no. 8, pp. $1284-1296,2010$

[17] S. Al-Hiddabi, J. Shen, and N. McClamroch, "A study of flight manoeuvres for the pvtol aircraft model," in Proc. of the American Control Conference, vol. 4, 1999, pp. 2727 -2731.

[18] G. Conte, C. Moog, and A. Perdon, Algebraic Methods for Nonlinear Control Systems, ser. Communications and Control Engineering. Springer, London, 2007.

[19] M. P., "An intrinsic sufficient condition for regular decoupling," Systems and control letters, vol. 20, no. 5, pp. 383-391, 1993, eng. 